\title{
CryoET Data Collection and Subtomogram Averaging Using emClarity
}

Tao Ni and Peijun Zhang

University of Oxford, Oxford, England, United Kingdom

Cryo-electron tomography (cryoET) can provide 3D reconstructions (i.e. tomograms) of pleomorphic objects such as organelles or cells in their close-to-native states. Subtomograms that contain repetitive structures can be further extracted and subjected to alignment, averaging and classification to improve resolution, and this process has become an emerging structural biology method referred as cryoET subtomogram averaging and classification (cryoSTAC). Compared to cryoEM single particle analysis (cryoEM SPA), cryoSTAC is still in its early stages. The greatest strength of cryoSTAC lies in in situ structure determination with 3D classification in native systems. It holds the potential to provide cellular landscapes of macromolecular complexes in near-atomic details with their spatial coordinates. Indeed, there are already a number of examples in which subnanometer resolution structures have been determined, and multiple functional states have been delineated in situ, allowing a direct connection between cellular function and the structure of macromolecular complexes. Here I will present the workflow of cryoET and cryoSTAC including specimen preparation, data collection, tomogram reconstruction and subtomogram averaging. Data collection in SerialEM and Tomo5 will be compared, followed by tilt series reconstruction using a number of available software packages, such as IMOD or Protomo, depending on the availability of fiducial marker. Lastly I will focus on the procedure of subtomogram averaging and classification using emClarity and give a few recent applications of cryoET and cryoSTAC in biological samples including: 1) atomic structure of the HIV-1 immature Gag particles to understand retrovirus assembly; 2) structure of in vitro reconstituted monolayer arrays of E. coli chemotaxis signalling complexes; 3) whole-cell lamella of cyanobacteria to understand the molecular organization of thylakoid membrane; 4) in situ structures of reovirus assembly intermediates in infected mammalian cell.

References

1. Zhang, P. Advances in cryo-electron tomography and subtomogram averaging and classification. Curr. Opin. Struct. Biol. 58, 249-258 (2019).

2. Mastronarde, D. N. Automated electron microscope tomography using robust prediction of specimen movements. J. Struct. Biol. 152, 36-51 (2005).

3. Hagen, W. J. H., Wan, W. \& Briggs, J. A. G. Implementation of a cryo-electron tomography tilt-scheme optimized for high resolution subtomogram averaging. J. Struct. Biol. 197, 191-198 (2017).

4. Mastronarde, D. N. \& Held, S. R. Automated tilt series alignment and tomographic reconstruction in IMOD. J. Struct. Biol. 197, 102-113 (2017).

5. Noble, A. J. \& Stagg, S. M. Automated batch fiducial-less tilt-series alignment in Appion using Protomo. J. Struct. Biol. 192, 270-278 (2015).

6. Himes, B. A. \& Zhang, P. emClarity: software for high-resolution cryo-electron tomography and subtomogram averaging. Nat. Methods 15, 955-961 (2018).

7. Schur, F. K. M. et al. An atomic model of HIV-1 capsid-SP1 reveals structures regulating assembly and maturation. Science 353, 506-508 (2016).

8. Cassidy, C. K. et al. CryoEM and computer simulations reveal a novel kinase conformational switch in bacterial chemotaxis signaling. Elife 4, 1193 (2015). 